\title{
Descripción del problema de dengue con enfoque de la determinación social de la salud en una comunidad: estudio de campo
}

\author{
*Cesar López García ${ }^{1,2}$, Ángel Francisco Betanzos Reyes ${ }^{1}$, María de la Luz Arenas Monreal ${ }^{3}$, Inés \\ Tenahua Quitı', María Luz De Ávila Arroyo² \\ ${ }^{1}$ Instituto Nacional de Salud Pública, Escuela de Salud Pública de México, Centro de investigación sobre \\ Enfermedades Infecciosas. México \\ ${ }^{2}$ Benemérita Universidad Autónoma de Puebla, Facultad de Enfermería. México \\ ${ }^{3}$ Centro de Investigaciones en Sistemas de Salud. México
}

\begin{abstract}
Cómo referenciar este artículo/
\end{abstract}
How to reference this article:

\begin{abstract}
López García C, Betanzos Reyes AF, Arenas Monreal ML, Tenahua Quitl I, De Ávila Arroyo ML. Descripción del problema de dengue con enfoque de la determinación social de la salud en una comunidad: estudio de campo. Mem. Inst. Investig. Cienc. Salud. 2019; 17(2): 6-15
\end{abstract}

\section{RES U M E N}

Este trabajo quiere contribuir con otra perspectiva a la solución del problema del Dengue en México. El objetivo fue determinar tipos de criadero de Aedes aegypti, condiciones de la vivienda e identificar dimensiones que determinen la enfermedad con enfoque integral social en la colonia centro de Mazatepec, Morelos. El estudio es de corte transversal descriptivo, se llevó a cabo en temporada de sequía (2015), se realizó colecta entomológica para identificar criaderos del vector, aplicación de Índice de Condición de Vivienda (ICV) y cuestionario con perspectiva de la Determinación Social de la Salud a 80 casas. Se detectaron 3,221 recipientes sin agua y 655 con agua, $25.34 \%$ tratables (tanques, tinacos), $9.46 \%$ controlables (botes y cubetas) y $4.7 \%$ diversos chicos, el ICV identificó 14 casas positivas (57.69\%-tratables), $47.5 \%$ de medio riesgo para crecimiento del vector, el $83.3 \%$ atribuyó el problema a condiciones de vida y posesión de recursos, $40.83 \%$ responsabilizó a la comunidad, falta de interés y mala organización. Las estrategias y políticas en salud deben de tomar en cuenta la perspectiva social y análisis de las comunidades, mejorar las condiciones de vivienda, trabajo y organización comunitaria para preservar la salud.

Palabras clave: epidemiología crítica, dengue, determinantes sociales, estudio transversal.

\section{Description of the dengue problem with a focus on the social determinants of health in a community: field study}

\section{A B S T R A C T}

This work aims to contribute to the solution to the dengue problem in Mexico with a different approach. The objectives of the research were to determine the types of hatcheries for Aedes aegypti and housing conditions, as well as to identify the dimensions that determine the disease with an integral social approach in the Colonia Centro of Mazatepec, Morelos, Mexico. This is a descriptive cross-sectional study

Fecha de recepción: junio 2019. Fecha de aceptación: julio 2019

*Autor correspondiente: Cesar López García. Instituto Nacional de Salud Pública. Escuela de Salud Pública de México. Centro de investigación sobre Enfermedades Infecciosas. Dirección: Universidad No. 655 Colonia Santa María Ahuacatitlán, Cerrada Los Pinos y Caminera C.P. 62100, Cuernavaca, Morelos. México. Email: cesar_asturiano@hotmail.com 
carried out during the dry season in 2015, an entomological collection was made to identify the vector hatcheries, the Housing Condition Index $(\mathrm{HCI})$ and a questionnaire were applied to establish the Social Determinants of Health for 80 houses. A total of 3,221 containers without water and 655 with water were detected, out of these $25.34 \%$ were tagged as manageable (water tanks), $9.46 \%$ as controllable (buckets and cans) and $4.7 \%$ as various small items. The HCI identified 14 positive houses ( $57.69 \%$ as manageable), $47.5 \%$ as medium risk for the development of the vector. Eighty three point three percent of the questionnaire participants attributed the problem to life conditions and resources property, $40.83 \%$ to the community, the lack of interest and disorganization as responsible. Health strategies and policies must take into account the social approach and analysis of the communities, improving housing, work and community organization conditions to preserve health.

Keywords: Critical epidemiology, dengue, social determinants, cross-sectional study.

\section{INTRODUCCIÓN}

En México las enfermedades transmitidas por vector han ocupado el gran interés de las autoridades de salud a nivel nacional, estatal y local y son consideradas como problema de salud pública. Entre ellos destacan las complicaciones ocasionadas por el virus del dengue que incluye 4 serotipos (DEN1, DEN2, DEN3 y DEN4), es trasmitido por el mosquito Aedes aegypti especie que vive en cercanía con humanos, en lugares donde las condiciones son propicias para su desarrollo, crecimiento y transmisión, principalmente en áreas urbanas tropicales ${ }^{(1)}$. En el Estado de Morelos se han presentado brotes importantes en diversos municipios con circulación DEN1 y DEN2, que favorecen la presencia y aumento de casos de fiebre hemorrágica por dengue $(\mathrm{FHD})^{(1,2)}$. En países con los niveles más altos de desigualdad social (índice de Gini), analfabetismo y habitantes que viven sin acceso a servicios de agua y saneamiento ${ }^{(3)}$ se ha reportado que el desarrollo del vector y transmisión de la enfermedad en una comunidad se debe a factores sociales y económicos; para contrarrestar el problema de salud, dentro de las estrategias para el control del dengue se implementa un enfoque clásico basado en una perspectiva epidemiológica de riesgo y biológica sobre el papel vectorial del Aedes aegypti que limita el alcance de un control efectivo ${ }^{(4,5)}$. Por esta razón ante la eventualidad de un brote o epidemia de dengue, se responsabiliza al mosquito como transmisor de la enfermedad.

Tal complejidad para abordar este esquema integral sostenible podría dilucidar el modelo de los determinantes sociales de la salud basado en identificar los factores de riesgo que padecen las personas de dengue, empero, éste modelo desarticula el proceso social-histórico del problema de salud en la comunidad ${ }^{(6)}$. El abordaje de las perspectivas epidemiológicas de riesgo y biológica, determinantes sociales de la salud y los programas de control del dengue, ha dado como resultado acciones desintegradas de las diferentes disciplinas científicas y de las necesidades de la comunidad; algunas de esas medidas como ejemplo son la eliminación del vector por medio de plaguicidas e incremento en conocimientos biomédicos, programas asistenciales y pasividad de la comunidad. El presente trabajo incluye una perspectiva complementaria para aportar una visión del problema de salud distinta, que incluya la identificación de ciertos procesos históricos de salud; la reflexión toma como marco conceptual la perspectiva de la epidemiología crítica de Salud Colectiva( ${ }^{(7,8)}$ y la Determinación Social de la Salud expuesta por Jaime Brehil ${ }^{(6,8)}$. Éste considera a la salud como un objeto multidimensional y crea tres principales dimensiones: sociedad, grupos e individuos. Cada dimensión tiene a su vez elementos protectores para mantener la salud: 1) la dimensión de la sociedad $(G)$ se integra por las relaciones de los sistemas y/o modelos económico, político, social y cultural históricamente determinados. Tiene como elemento protector la solidaridad, sustentabilidad y bioseguridad; 2) la dimensión de grupos (P) se caracteriza por los modos de vivir de la sociedad, teniendo como elemento protector la calidad y disfrute de los bienes de consumo del grupo, organización y acciones en beneficio a la salud y las condiciones del trabajo; 3) la dimensión individual (S) se integra por los procesos del estilo de 
vida, como elemento protector, integra la capacidad personal para tener una alimentación adecuada, descanso, vivienda, acceso y calidad de los servicios ${ }^{(6,8)}$. El desequilibrio y los procesos no saludables en todas las dimensiones pueden determinar cómo enfermar en un momento dado en la comunidad.

Por lo anterior esta investigación plantea determinar los tipos de criadero de Aedes aegypti, condiciones de la vivienda e identificar las dimensiones que determinen la enfermedad con un enfoque integral de la determinación social de la salud; en la dimensión general se incluye el conocimiento de su entorno económico y político, las condiciones de vivir y situación laboral; dimensión de grupos, la problemática del dengue en su comunidad, colaboración y participación de la comunidad y por último dimensión individual, conocimientos básicos biomédicos de la enfermedad del dengue.

\section{MATERIALES Y MÉTODOS}

Área de estudio. El estudio se realizó en la localidad colonia Justo Sierra del Municipio de Mazatepec estado de Morelos (NN18 $43^{\prime} 59^{\prime \prime} \mathrm{N} 99^{\circ} 22^{\prime} 01^{\prime \prime O}$ ) con una altitud de 960 msnm y 9,456 habitantes. La distancia de la localidad a la capital del estado es de $41 \mathrm{~km}$. Estas localidades forman parte de siete ciudades de alto riesgo de transmisión de dengue en las zonas urbanas del sur y del este de Morelos. El clima de la localidad es de tipo cálido subhúmedo, caluroso y tropical, con inviernos poco definidos y con mayor sequía en el otoño, invierno a principios de primavera, la temperatura es de $23.60^{\circ} \mathrm{C}$ y la precipitación pluvial es de $1,194 \mathrm{~mm}^{3(9,10)}$.

Diseño del estudio y tamaño de muestra. Se llevó a cabo en temporada de sequía (febrero a mayo del 2015). El muestreo se realizó por aleatorización simple al utilizar el Área Geoestadística Básica (AGEB) como unidad ${ }^{(11)}$. Se revisaron 20 casas por cada AGEB; quedando un total de 80 casas $^{(11)}$. Se ubicó a la periferia del AGEB y desde allí se seleccionó una casa cada lado de la calle y dejando cinco casas por cada casa inspeccionada, hasta completar 20 por AGEB (Figura 1). Se explicó a los participantes el objetivo del estudio para inspeccionar y colectar muestras entomológicas y así determinar tipos de criadero de Aedes aegypti y las condiciones de la vivienda, además de aplicar el cuestionario para identificar las dimensiones que determinen la enfermedad del dengue. Las colectas se llevaron a cabo entre las 8:00 y las 16:00 horas por equipos conformados por dos personas especializadas en actividades de entomología del programa de dengue del estado y dos personas del municipio.

Colectas entomológicas y de aplicación de instrumentos. La aplicación del cuestionario contó con 35 reactivos de respuesta cerrada, el formato se aplicó a jefes de familia al momento de la inspección de la casa para la aplicación del cuestionario previamente construido por expertos con fundamento de la determinación social de la salud $^{(6,8)}$. Se realizó la inspección activa de larvas y pupas de mosquitos en todos los recipientes potenciales como tratables (tanques, llantas, pilas, piletas, tinajas, tinacos, pozos, aljibes, sanitarios y diversos grandes), controlables (botes y cubetas, floreros y plantas acuáticas, tinas, bebedores de animales, aires lavados, macetas y macetones) y diversos chicos (latas, botellas, trastes y tapas) para el desarrollo de criaderos de acuerdo a los instrumentos de Entomológica EA-1 ${ }^{(11)}$. Se consideró positivo al menos uno larva o pupa en el recipiente inspeccionado fuera de las casas, se describieron las abundancias de pupas y larvas de Ae. aegypti entre los tipos de criaderos encontrados en las viviendas por contribución porcentual definida como el número de pupas y larvas en el total de recipientes de una categoría, dividido entre el número total de pupas y larvas en todos los recipientes ${ }^{(12)}$. En la variante Índice de Casa Positiva (ICP) se encuentran larvas o pupas en cualquiera de los recipientes con agua explorados. Las variables consideradas para el análisis fueron: el número total de recipientes por categoría $(n)$, recipientes con agua (RA), número absoluto de pupas, número absoluto de larvas, recipientes con agua positivos a larvas de Ae. aegypti (RP) y recipientes positivos a pupas de Ae. aegypti (RPPU). En el Índice de Condición de Vivienda $(\mathrm{ICV})^{(11)}$ se tomaron como variables: apariencia de la casa (oquedades y apariencia global de la casa), ventanas, puertas, recipientes con agua dentro o fuera de la casa, sombra del patio y suciedad del patio con el fin de relacionar las condiciones de vivir. 
López García et al Descripción del problema de dengue con enfoque de la determinación social...

Se utilizó Software Stata 12. El protocolo fue aprobado por la Comisión de Ética del Instituto Nacional de Salud Pública.

\section{RESULTADOS}

Tipología de los recipientes: De las 80 casas visitadas, se revisó el interior y exterior de la vivienda en época de sequía de las cuales 14 fueron positivas a larvas y pupas. Se identificaron recipientes con agua de los cuales 655 recipientes fueron artificiales con un promedio de $8.18(D E=6.25)$ por casa localizados con puntos rojos como se muestra en la Figura 1; con un Índice de Casa Positiva (ICP) a larvas de 29.5, se registraron 3,221 recipientes sin agua con un promedio por casa de 40.26 $(D E=20.22)$ recipientes (Tabla 1$)$.

Tabla 1: Índice de condición de vivienda, riesgo y positividad en las casas. n: 80

\begin{tabular}{|c|c|c|c|c|}
\hline Características & & $n$ & $\%$ & IC $95 \%$ \\
\hline \multirow[t]{3}{*}{ ICV } & Bajo riesgo & 9 & 11.25 & $.02-.15$ \\
\hline & Medio riesgo & 38 & 47.50 & $.36-.58$ \\
\hline & Alto riesgo & 33 & 41.25 & $.30-.52$ \\
\hline \multirow{4}{*}{ Positividad y el ICV } & Bajo riesgo & 3 & 21.43 & $.00-.10$ \\
\hline & Medio riesgo & 5 & 35.71 & $.02-.13$ \\
\hline & Alto riesgo & 6 & 42.86 & $.02-.15$ \\
\hline & Total de positivas & 14 & & \\
\hline \multirow{6}{*}{$\begin{array}{l}\text { Apariencia Global de la casa } \\
\text { 1.(Oquedades, aberturas o } \\
\text { huecos en la pared) } \\
\text { 2.Ventanas, puertas } \\
\text { 3.Recipientes con agua dentro } \\
\text { o fuera de la casa }\end{array}$} & & 25 & 31.25 & $.21-.42$ \\
\hline & Bajo riesgo & & & \\
\hline & & 30 & 37.50 & $.26-.49$ \\
\hline & Medio riesgo & & & \\
\hline & & 25 & 31.25 & $.21-.42$ \\
\hline & Alto riesgo & & & \\
\hline \multirow[t]{3}{*}{ Sombra del patio } & $<25 \%$ Bajo riesgo & 22 & 27.50 & $.18-.38$ \\
\hline & $26-50 \%$ Medio riesgo & 24 & 30 & $.20-.41$ \\
\hline & $>50 \% \quad$ Alto riesgo & 34 & 42.50 & $.31-.54$ \\
\hline \multirow[t]{3}{*}{ Suciedad del patio } & Limpio & 34 & 2.50 & $.31-.54$ \\
\hline & Algo sucio (pasto, basura) & 25 & 31.25 & $.21-.42$ \\
\hline & $\begin{array}{l}\text { Sucio y con recipientes con } \\
\text { agua }\end{array}$ & 21 & 26.25 & $.17-.37$ \\
\hline
\end{tabular}

Fuente: elaboración propia

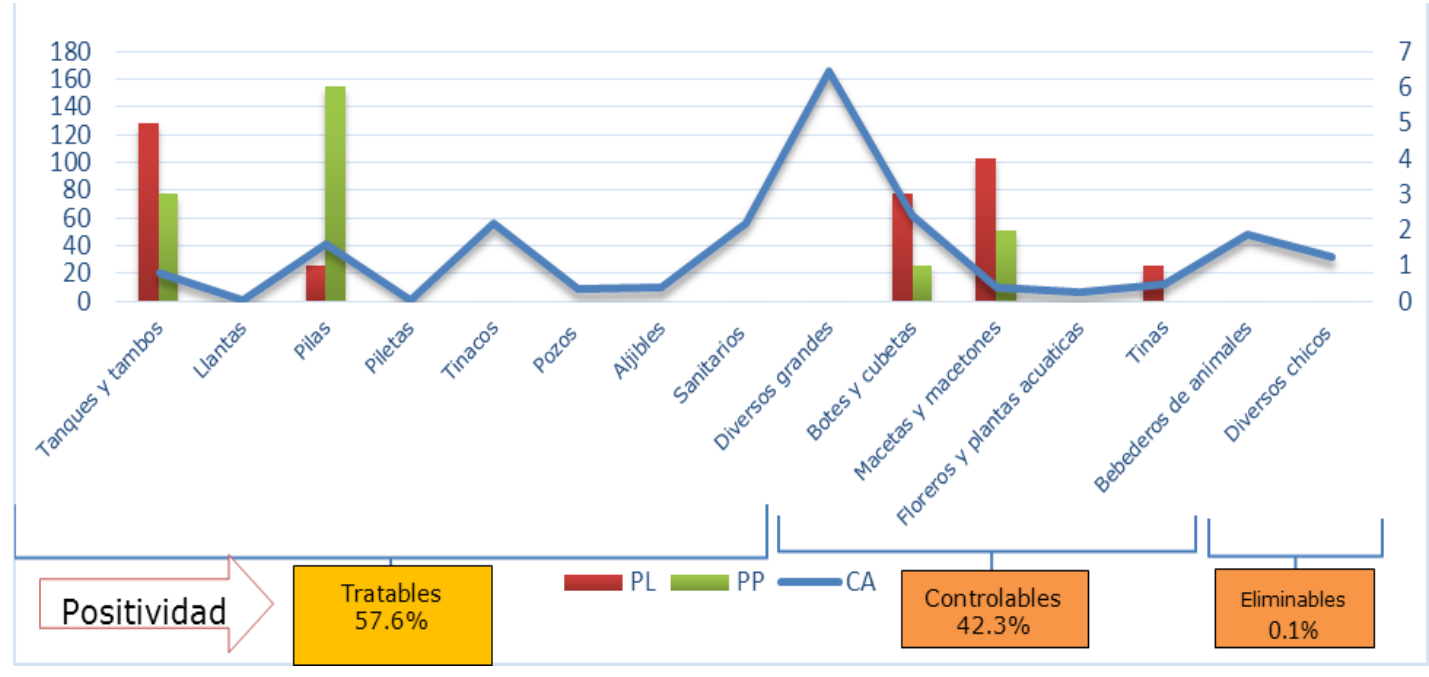

Figura 1: Tipología y positividad de los recipientes en la colonia Justo Sierra, Mazatepec, Morelos. 
Categorización de los recipientes: se encontraron 3,321 recipientes sin agua (Figura 2).

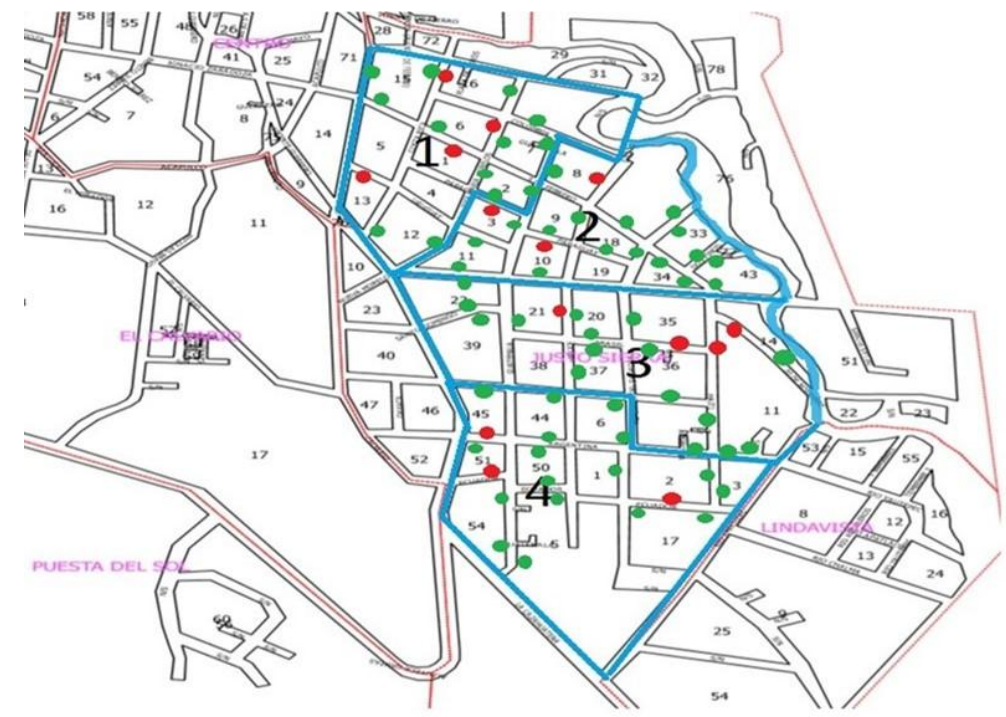

Fuente: elaboración propia, identificación de casas positivas con puntos de color rojo.

Figura 2: Localización geográfica y distribución de las casas en la Colonia Justo Sierra, Mazatepec, Morelos.

-Tratables comprende: tanques, llantas, pilas, piletas, tinajas, tinacos, pozos, aljibes, sanitarios y diversos grandes. Estos representaron $15.62 \%$ con mayor número de recipientes en diversos grandes con $7.2 \%$.

-Controlables comprende: botes y cubetas, macetas y macetones, floreros y plantas acuáticas, tinas, bebedores de animales, aires lavados. Estos representaron el $42.8 \%$ con mayor número de recipientes en macetas y macetones $32 \%$.

-Diversos chicos (eliminables): latas, botellas, trastes y tapas. Estos representaron el $8.1 \%$ de los recipientes totales sin agua.

De los 655 recipientes con almacenamiento de agua los tratables representaron el $54.8 \%$, los recipientes controlables el $20.9 \%$ y los diversos chicos con el $4.7 \%$ (Figura $1)$.

-Tratables con agua y mayor proporción de recipientes en diversos grandes con $25.3 \%$ -Controlables con agua y mayor proporción de recipientes en botes y cubetas $9.4 \%$.

Índice de Condición de Vivienda (ICV): De las 14 casas positivas identificadas con relación con el ICV, el $42.8 \%$ tenía alto riesgo, $35.7 \%$ de medio y $21.4 \%$ de bajo riesgo para el desarrollo del vector. De las 80 casas visitadas el $47.5 \%$ se encontraba en mediano riesgo, caracterizada por tener $50 \%$ mayor sombra de patio que propicia la formación de criaderos, sin embargo, se identificó que el $34 \%$ se encontraba en patio limpio (Tabla 1).

Características generales del cuestionario: se excluyeron 20 cuestionarios incompletos $(n=60)$, edad promedio de 50 años, ( $D E=15.43)$ (IC95\% 45.76-53.73). En cuanto a sexo y jefe de familia, predominó la mujer con $81.6 \%$ y $76.6 \%$ respectivamente (Tabla 1 ).

Dimensión de la sociedad (G): En relación a la percepción de la comunidad el $75 \%$ mencionó que hay dengue en todo el mundo, el $55 \%$ en países en vías de desarrollo y un $44.8 \%$ asignó la culpabilidad a la comunidad. La perspectiva de quienes se enferman de dengue con relación al desarrollo económico del país lo atribuían a las condiciones de vida y posesión de recursos necesarios para vivir en un $83.33 \%$, sin embargo, el $98.31 \%$ mencionó que si tuvieran mejores condiciones laborales ayudaría a mejorar las condiciones de su vivienda, con la posibilidad de disminuir enfermar por dengue (Tabla 2). 
López García et al Descripción del problema de dengue con enfoque de la determinación social...

Tabla 2: Dimensión de la sociedad (G). n: 60

\begin{tabular}{|c|c|c|c|c|}
\hline \multicolumn{2}{|l|}{ Características } & \multirow{3}{*}{$\begin{array}{l}n \\
2 \\
33\end{array}$} & \multirow{2}{*}{$\begin{array}{l}\% \\
3.33\end{array}$} & \multirow{2}{*}{$\begin{array}{c}\text { IC 95\% } \\
.004-.11\end{array}$} \\
\hline Mayor dengue en países & -Países desarrollados & & & \\
\hline desarrollados o en vías & -Países en vías de desarrollo & & 55 & $.41-.67$ \\
\hline \multirow[t]{2}{*}{ de desarrollo } & -Igual & 23 & 38.33 & $.26-.51$ \\
\hline & -Otro & 2 & 3.33 & $.004-.11$ \\
\hline \multirow{4}{*}{$\begin{array}{l}\text { ¿Por qué cree usted esa } \\
\text { respuesta? }\end{array}$} & -Gobierno y Empresas & 12 & 20.68 & $.10-.32$ \\
\hline & -La comunidad & 26 & 44.83 & $.30-.56$ \\
\hline & -El clima y situación geográfica & 13 & 22.41 & $.12-.34$ \\
\hline & -Otros & 7 & 12.08 & $.04-.22$ \\
\hline \multirow{3}{*}{$\begin{array}{l}\text { Responsables de las } \\
\text { condiciones de vivir }\end{array}$} & -La comunidad & 51 & 85 & $.73-.92$ \\
\hline & -El gobierno y las empresas & 6 & 10 & $.03-.20$ \\
\hline & -Otro & 2 & 5 & $.0004-.08$ \\
\hline \multirow{4}{*}{$\begin{array}{l}\text { ¿Por qué cree esa } \\
\text { respuesta? }\end{array}$} & -Las condiciones laborales & 19 & 31.67 & $.20-.44$ \\
\hline & -Falta de interés y organización & 30 & 50 & $.36-.63$ \\
\hline & -Corrupción & 1 & 1.67 & $.0004-.08$ \\
\hline & -Otro & 10 & 16.66 & $.03-.20$ \\
\hline \multirow[t]{6}{*}{ Problemática del dengue } & -Las condiciones del trabajo & 3 & 5.08 & $.01-.13$ \\
\hline & $\begin{array}{l}\text {-Mala organización y falta de } \\
\text { interés entre la comunidad y }\end{array}$ & & & \\
\hline & autoridades & 34 & 56.66 & $.43-.69$ \\
\hline & -Contaminación & 16 & 27.12 & $.16-.39$ \\
\hline & -Pobreza & 5 & 8.47 & $.02-.18$ \\
\hline & -Otro & 1 & 2.67 & $.0004-.08$ \\
\hline \multirow{3}{*}{$\begin{array}{l}\text { Limitante del control del } \\
\text { dengue la comunidad }\end{array}$} & -No hay suficientes plaguicidas & 19 & 32.20 & $.20-.44$ \\
\hline & $\begin{array}{l}\text {-No hay colaboración de la } \\
\text { comunidad }\end{array}$ & 23 & 38.98 & $.26-.51$ \\
\hline & $\begin{array}{l}\text {-Deficiencia en los servicios } \\
\text { básicos }\end{array}$ & 9 & 15.25 & $.07-.265$ \\
\hline
\end{tabular}

Dimensión de grupos $(P)$ : Para contribuir al problema del dengue el $91.53 \%$ consideró necesario una organización de la comunidad que ayude a controlar el problema del dengue, además el $70 \%$ estaba dispuesto a participar en un comité de salud comunitario. La población responsabilizó a la comunidad en un $85 \%$ sus condiciones de vida y cuando respondió a ¿Por qué cree esa respuesta? afirmó que era debido a las condiciones laborales, falta de interés y organización, así como por la contaminación ambiental (27.1\%). Para el control del dengue, el $91.53 \%$ mencionó una organización de la comunidad para mitigar el problema, y además que participaría en los comités de salud comunitario e independientemente de las autoridades (Tabla 3).

Tabla 3: Dimensión de grupos (P). n: 60

\begin{tabular}{llccc}
\hline Características & & $n$ & $\%$ & IC 95\% \\
\hline Organización de la comunidad para controlar el & $\mathrm{Si}$ & 55 & 91.66 & $.79-.96$ \\
problema del dengue & $\mathrm{No}$ & 5 & 8.33 & $.02-.18$ \\
Comité de Salud comunitario custed & $\mathrm{Si}$ & 42 & 70 & $.56-.81$ \\
participaría? & $\mathrm{No}$ & 18 & 30 & $.18-.43$ \\
Colaboración en las acciones de control del & $\mathrm{Si}$ & 56 & 93.33 & $.83-.98$ \\
dengue independientemente de las autoridades & $\mathrm{No}$ & 4 & 6.67 & $.01-.16$ \\
\hline Fuente: elaboración propia & & & &
\end{tabular}

Dimensión individual (S): En cuanto al conocimiento de la transmisión del dengue por picadura de un mosquito, $88.33 \%$ declaró saberlo. Por último, en cuanto al conocimiento de la enfermedad del dengue, el $64,41 \%$ identificó que es una enfermedad, poseía conocimientos del dengue clásico y hemorrágico, conocía los síntomas y la transmisión por mosquito maduro, sin embargo, no tenía conocimiento de las fases acuática o inmaduras del vector (Tabla 4). 
Tabla 4: Dimensión individual (S). n: 60

\begin{tabular}{llccc}
\hline Características & & $\boldsymbol{n}$ & $\mathbf{\%}$ & IC 95\% \\
\hline Conocimiento del dengue & Una enfermedad & 38 & 64.41 & $.49-.75$ \\
& Un virus & 7 & 11.86 & $.04-.22$ \\
& Un mosquito/maromero & 11 & 18.64 & $.07-.26$ \\
& Otro & 3 & 5.09 & $.01-.13$ \\
\hline Tipos de enfermedad del dengue & Dengue clásico & 35 & 61.40 & $.44-.70$ \\
& Dengue hemorrágico & 10 & 17.54 & $.08-.28$ \\
& Ambos & 45 & 78.94 & $62-.85$ \\
& No se & 12 & 21.05 & $.10-.32$ \\
\hline Principal síntoma del dengue & Fiebre & 21 & 35 & $.23-.48$ \\
clásico & Cefalea & 9 & 15 & $.07-.26$ \\
& Astenia y adinamia & 26 & 43.33 & $.30-.56$ \\
& Otro & 4 & 6.67 & $.00-.08$ \\
\hline Conocimiento de la larva del & Si & 23 & 38.33 & $.26-.51$ \\
mosquito transmisor del dengue & No & 37 & 61.67 & $.48-.73$ \\
\hline Conocimiento de la pupa del & Si & 7 & 11.67 & $.04-.22$ \\
mosquito transmisor del dengue & No & 53 & 88.33 & $.77-.95$ \\
\hline Fuente: & & & &
\end{tabular}

\section{DISCUSIÓN}

La comunidad tiene un riesgo de trasmisión del dengue de acuerdo con los resultados de la tipología de los recipientes positivos a larvas y pupas, además reflejados en los índices entomológicos (ICP, IRP, IB) posiblemente por la escases de agua y almacenamiento en recipientes controlables (tambos y botes/cubetas) en estudios realizados en otras áreas similares a Morelos (Cuautla, Jojutla y Tlaquiltenango ${ }^{(13)}$, en Cuautla, Morelos y Mérida Yucatán ${ }^{(15)}$ los resultados fueron variables con positividad en los recipientes tratables y diversos chicos respectivamente, sin embargo, en dos municipios restantes de Morelos los resultados fueron similares a los de este estudio con mayor porcentaje en controlables en condiciones climatológicas en temporada seca ${ }^{(13,14)}$.

En cuanto al índice de condición de la vivienda se evidenció riesgo alto y medio para las casas encontradas además de considerar al patio como reservorio que propicia las condiciones óptimas para el desarrollo del vector, no se encontraron datos disponibles en otros estudios, sin embargo, en estudios relacionados con las variables de la condición de la vivienda, los resultados son congruentes en presencia de patio con sombra parcial, vegetación y de árboles así como con la mala higiene ${ }^{(16)}$. En un estudio en Costa Rica las variables de importancia fueron la temperatura, la altitud, el índice de pobreza humana e incluyeron además índice de la vivienda. Esta última debido a que las condiciones desfavorables de la vivienda como el estado de la vivienda son un factor para el desarrollo del vector entre otras ${ }^{(17)}$. Otro estudio en México relaciona las condiciones de la vivienda con la disposición de residuos fuera de la recolección municipal significativamente para el desarrollo del vector ${ }^{(18)}$.

Respecto a la percepción de la dimensión general (G) se halló la consideración sobre el conocimiento del dengue en todo el mundo, consideración en países en vías de desarrollo, lo que concuerda con un estudio, que la enfermedad está en todos lados, es un riesgo y genera alerta, además es una epidemia que afecta a todos y existe falta de control sobre la misma ${ }^{(19)}$, es un problema de salud pública endémico en algunas regiones de las Américas ${ }^{(20)}$.Otro estudio encontró que la atribución de la responsabilidad de prevenir es de otras personas u organismos públicos, con similitud a los resultados de este estudio de culpar a la comunidad ${ }^{(19)}$. La perspectiva de quienes se enferman de dengue con relación al desarrollo económico del país resultó que lo atribuían a las condiciones de vida y posesión de recursos necesarios para vivir, no encontramos estudios similares bajo esta perspectiva, empero, algunas variables tomadas en cuenta fueron consecuencias laborales, ya que se menciona la falta de trabajo y dinero, la incapacidad laboral y el perjuicio al bienestar económico de la familia ${ }^{(19)}$, esto coincide o concuerda con esta investigación en la que dijeron que si tuvieran mejores condiciones laborales ayudaría a mejorar las condiciones de su vivienda, con la posibilidad de disminuir enfermar por dengue. 
En la percepción de la dimensión grupal ( $P$ ) la comunidad estaba dispuesta a colaborar con o sin el municipio, y tomar las acciones en salud con actores clave de la comunidad, trabajadores de la salud y municipales, sin embargo, también en nuestro estudio se observó la falta de organización, el culpar a la comunidad, la deficiencia en los servicios básicos, la desconfianza hacia sus autoridades, estrategias poco efectivas y corrupción que interfieren en las acciones de prevención del dengue. Estudios similares mostraron que la participación social es necesaria para la prevención, que existe temor al contagio y preocupación por los enfermos y por las fugas de aguas servidas, existe una falta de control por parte de las autoridades lo que ocasiona alarma social y muerte, además de la necesidad de acceso a servicios básicos de saneamiento ${ }^{(17,19,21,22)}$.

Por otro lado, la población en edades adultas respecto a la dimensión individual (S) percibe el problema del dengue en aspectos superficiales tanto en la enfermedad, la transmisión y algunas acciones de prevención. Las intervenciones educativas enfocadas al dengue se han establecido como el incremento de los conocimientos en las poblaciones de estudio mediante capacitaciones ${ }^{(3,6,8)}$. Por ejemplo: el conocimiento de la enfermedad, tipo de enfermedad clínica, transmisión, criaderos del vector y medidas preventivas, posiblemente la población estudiada tuvo esa información, en contraste con un estudio que menciona que el conocimiento de la información es confusa e inclusive insuficiente, además tomar como medida principal de control el uso excesivo de la fumigación ${ }^{(18,23)}$.

Ante la escasez de investigación social sobre las enfermedades infecciosas, existe una brecha en las colaboraciones científicas con el área biomédica y social, esta necesidad de diálogo de acuerdos para la explicación de los fenómenos en salud aun es pobre, es por ello que la enfermedad es vista solamente desde la perspectiva biomédica, este estudio aporta este acercamiento a los factores sociales desde la perspectiva de la determinación social de la salud y la enfermedad del dengue, este abordaje es visto también en otros estudios con diferentes temas de salud ${ }^{(20,24-26)}$.

Es fortalecida la aportación científica y reforzamientos de paradigmas que dan explicación de los fenómenos en salud, la observación de lo complejo requiere de la contribución de las distintas miradas académicas surgidas de los teóricos, operativos y experiencia de las colectividades. Se requiere integrar elementos y aportaciones críticas del sistema social imperante de distintos enfoques, paradigmas, teorías, investigaciones y opiniones, para la contribución a la investigación científica ${ }^{(24,25,27-29)}$.

El problema del dengue desde la perspectiva de la determinación social de la salud comprende más allá del control y eliminación del vector, la indagación de la situación económica, política y laboral de las personas, modos de vivir, consumo, colaboración y participación comunitaria, además de la degradación de la salud como corrupción y acciones de salud superficiales como control con plaguicidas. La inclusión de las variables antes expuestas en estudios epidemiológicos podría proporcionar datos para el cambio de las estrategias de control de enfermedades transmitidas por vector y posiblemente para otras enfermedades infecciosas. Los resultados demuestran la necesidad de contemplar la realidad de la comunidad, ya que existen vacíos en los procesos de salud-enfermedad, sin embargo, esta perspectiva se debe complementar con estudios cualitativos y otros diseños de investigación que posiblemente determinen que la población enferme o padezca daños a la salud.

\section{Agradecimientos}

Agradecemos a los habitantes de la Colonia Justo Sierra, Mazatepec Morelos y al Instituto Nacional de Salud Pública por la aportación científica y colectiva.

Fuentes de financiación: El trabajo fue financiado con fondos propios de los autores.

Conflicto de intereses: Los autores no declaran conflictos de intereses. 


\section{REFERENCIAS BIBLIOGRÁFICAS}

1. Vázquez-Pichardo $M$, Rosales-Jiménez C, Núñez-León A, Rivera-Osorio $P$, De La Cruz-Hernández S, Ruiz- López $A$, et al. Serotipos de dengue en México durante 2009 y 2010. Bol Med Hosp Infant Mex 2011; 68(2):103-110.

2. Organización Panamericana de la Salud. Últimos adelantos técnicos en la prevención y el control del dengue en la Región de las América. 28 y 29 de mayo del 2014. Washington, D.C. EUA. Consultado: agosto 2015 Disponible en:

http://www.paho.org/hq/index.php?opt ion $=$ com_topics \&view $=$ article\&id $=1 \&$ It emid $=40734$ \&lang $=$ es

3. Granda Edmundo. A qué Ilamamos salud colectiva, hoy. Rev Cubana Salud Pública. Abr.-Jun. 2004 v.30 n.2 (revista en la Internet). Disponible en: http://scielo.sld.cu/scielo.php?script $=$ s ci_arttext\&pid=S086434662004000200009\&lng=es.

4. NORMA Oficial Mexicana NOM-032SSA2-2002, Para la vigilancia epidemiológica, prevención y control de enfermedades transmitidas por vector. Lunes 21 de julio de 2003. Diario Oficial de la Federación. Disponible: http://dof.gob.mx/nota_detalle.php?co digo $=5357366 \&$ fecha $=22 / 08 / 2014$ Secretaría de Salud. Guía para la Participación Comunitaria para la Prevención y Control del Dengue. México; 2013. p. 85.

5. EIbenschutz-Hartman C, TamezGonzález S, González-Guzmán R. ¿Determinación social o determinantes sociales de la salud? Primera Edición. Editorial Abate Faria. Universidad Autónoma Metropolitana 2011.

6. Breilh Paz y Miño J. Epidemiologia economía política y salud. Séptima edición. Ecuador Quito. Corporación Editorial Nacional. 2010.

7. Firpo de Souza Porto M, Ferreira da Rocha D, Finamore R. Saúde coletiva, território e conflitos ambientais: bases para um enfoque socioambiental crítico. Ciência \& Saúde Coletiva 2014, 19(10):4071-80.

8. Breilh J. La determinación social de la salud como herramienta de transformación hacia una nueva salud pública (salud colectiva). Rev. Fac. Nac. Salud Pública2013; 31(supl 1): S13-S27.

9. INEGI. Instituto Nacional de Estadística y Geografía. 2014. Consultado: octubre 2014. Disponible: http://www.inegi.org.mx
10. Hernández Ávila M. Epidemiología Diseño y análisis de estudio. Editorial Médica Panamericana S.A. de C.V. Hegel 141 2do piso, Chapultepec Morales C.P. 11570, México, D.F. 2007.

11. Centro Nacional de Programas Preventivos y Control de Enfermedades. Guía metodológica de estudios entomológicos para fase larvaria y pupal. CENAPRECE, 2013. Disponible

en: http://www.cenaprece.salud.gob.mx/pr ogramas/interior/vectores/dengue/guia s_operativas.html

12. Tapia-Conyer R, Méndez-Galván J, Burciaga-Zúñiga P. Community participation in the prevention and control of dengue: the patio limpio strategy in México. Journal Pediatric Child Health 2012; 32(1):10-13

13. Villegas-Trejo A, Che-Mendoza A, González-Fernández M, Guillermo-May G, González-Bejarano $\mathrm{H}$, DzulManzanilla $F$, et al. Control enfocado de Aedes aegypti en localidades de alto riesgo de transmisión de dengue en Morelos, México. Salud Pública de México marzo-abril de 2011; 53(2).

14. Arredondo-Jiménez JI, Valdez-Delgado KM. Aedes aegypti pupal/demographic surveys in southern Mexico: consistency and practicality. Ann Trop Med Parasitol 2006; 1:S17- S32.

15. Barrera-Pérez $M A$, Pavía-Ruz $N$, Mendoza-Mézquita JE, Torres-Arcila N, Hernández-Hernández R, CastroGamboa F, et al. Control de criaderos de Aedes aegypti con el programa Recicla por tu bienestar en Mérida, México. Salud Pública Mex 2015; 57:201-10.

16. Lazcano JAB, Marquetti M del C, Portillo $R$, Rodriguez $M M$, Suárez $S$, Leyva $M$. Ecological factors linked to the presence of Aedes aegypti larvae in highly infested areas of Playa, a municipality belonging to Ciudad de La Habana, Cuba. Revista Panamericana de Salud Pública (Internet). 2006 Jun (cited 2019 May 13);19(6):379-84. Available from: https://www.proxydgb.buap.mx:2057/l ogin. aspx ?direct $=$ true $\& d b=\mid$ th $\& A N=218$ 79788\&lang =es\&site $=$ ehost-live

17. Mena N, Troyo A, Bonilla-Carrión R, Calderón-Arguedas Ó. Factores asociados con la incidencia de dengue en Costa Rica. Revista Panamericana de Salud Pública (Internet). $2011 \mathrm{Apr}$ (cited 2019 May 13);29(4):234-42. Available from: https://www.proxydgb.buap.mx:2057// 
López García et al Descripción del problema de dengue con enfoque de la determinación social...

ogin.aspx?direct $=$ true $\& d b=a 9 h \& A N=62$ 955872\&lang=es\&site=ehost-live

18. Chuc S, Hurtado-Díaz M, Schilmann A, Riojas-Rodríguez $\mathrm{H}$, Rangel $\mathrm{H}$, González-Fernández MI. Condiciones locales de vulnerabilidad asociadas con dengue en dos comunidades de Morelos. Salud Publica Mex 2013; 54: 170-8

19. Torres López TM, Guerrero Cordero JL, Salazar Estrada JG. Dimensiones culturales del dengue que favorecen 0 dificultan su prevención en México. Rev Panam Salud Pública. 2012; 31(3):197-203.

20. Organización Mundial de la Salud. Dengue y dengue grave. 15 de abril de 2019. Disponible en: https://www.who.int/es/newsroom/fact-sheets/detail/dengue-andsevere-dengue

21. Kourí G, Pelegrino JL, Munster BM, Guzmán MG. Sociedad, economía, inequidades y dengue. Revista Cubana de Medicina Tropical (Internet). 2007 Sep (cited 2019 May 13); 59 (3):1-12. Available from: https://www.proxydgb.buap.mx:2057/I ogin.aspx?direct $=$ true $\& d b=\mid$ th $\& A N=317$ 93327\&lang $=$ es\&site $=$ ehost-live

22. Martin Giraldo-Hurtado T, Paola Álvarez-Betancur J, Parra-Henao G. Factores asociados a la infestación domiciliaria por Aedes aegypti en el corregimiento el Manzanillo, municipio de Itagüí (Antioquia) año 2015. Revista Facultad Nacional de Salud Pública [Internet]. 2018 Jan (cited 2019 May 13); 36(1):34-44. Available from:

https://www.proxydgb.buap.mx:2057// ogin. aspx?direct $=$ true $\& d b=\mid$ th $\& A N=129$ 800376\&lang=es\&site =ehost-live

23. Gutiérrez C, Montenegro Idrogo JJ. Conocimiento sobre dengue en una región endémica de Perú. Estudio debase poblacional. Acta Med Peru. 2017;34(4):283-8.
24. Arredondo Armando. Análisis y Reflexión sobre Modelos Teóricos del Proceso Salud-Enfermedad. Cad. Saúde Públ., Rio de Janeiro, jul/set, 1992, 8 (3): 254-61.

25. Harnecker Marta. "Los conceptos elementales del materialismo histórico", $6^{\circ}$ ed. México, España, Argentina, Siglo XXI Editores, 19852001.

26. González Llaca E. Corrupción Patología colectiva. Primera edición, México, Editorial Instituto Nacional de Administración Pública, 2005.

27. Firpo de Souza Porto M, Ferreira da Rocha D, Finamore R. Saúde coletiva, território e conflitos ambientais: bases para um enfoque socioambiental crítico. Ciência \& Saúde Coletiva 2014, 19(10):4071-80.

28. Pérez-Chacón $D$, Sánchez.Valdés $L$, Castro-Peraza $M$, López-Mc Bean $M$, Freyre-Hechavarría $B$, Inerarity-Rojas $C$ et al. Práctica-teoría-práctica transformada de una experiencia de participación comunitaria en el control de Aedes aegypti. Rev Cubana Med Trop, 2009; 61(3):282-7

29. Carmona Fonseca J, Correa AM. Determinación social de la desnutrición y el retardo psicomotor en preescolares de Urabá (Colombia). Un análisis con la Epidemiología Crítica. Rev. Fac. Nac. Salud Pública 2014; 32(1): 40-51. 